\title{
Interactions Between Sodium Dodecyl Sulfate Micelles and Peptides During Matrix-Assisted Laser Desorption/Ionization Mass Spectrometry (MALDI-MS) of Proteolytic Digests
}

\author{
Rama Tummala, Kari B. Green-Church* and Patrick A. Limbach \\ Rieveschl Laboratories for Mass Spectrometry, Department of Chemistry, University of Cincinnati, \\ Cincinnati, Ohio, USA
}

\begin{abstract}
Although sodium dodecyl sulfate (SDS) is routinely used as a denaturing agent for proteins, its presence is highly detrimental on the analysis of peptides and proteins by mass spectrometry. It has been found, however, that when SDS is present in concentrations near to or above its critical micelle concentration (CMC), improvements in the matrix-assisted laser desorption/ ionization mass spectrometry (MALDI-MS) analysis of peptide mixtures or hydrophobic proteins are obtained. To elucidate possible explanations for such improvements, here we have undertaken a study examining the effect of SDS micelles on peptide mixtures. Fluorescently labeled peptides were used as probes to determine whether hydrophobic or hydrophilic peptides interact exclusively with SDS micelles. In addition, four globular proteins were digested with trypsin and then various amounts of SDS were added before MALDI mass spectrometry. To examine the role of mixture complexity on the mass spectral results, the tryptic digest of bovine serum albumin was also fractionated according to hydrophobicity before SDS treatment. Results from these experiments suggest that micelle-peptide interactions increase peptide-matrix cocrystallization irrespective of analyte hydrophobicity. As these studies were performed using the dried-droplet method of sample spotting, the presence of micelles is also hypothesized to reduce Marangoni effects during the crystallization process. (J Am Soc Mass Spectrom 2005, 16, 1438-1446) (C) 2005 American Society for Mass Spectrometry
\end{abstract}

$\mathrm{T}$ The advantage of using matrix-assisted laser desorption/ionization mass spectrometry (MALDIMS) for peptide mass fingerprinting is that it allows for the rapid identification of proteins with minimal sample clean-up before the analysis. To obtain stable and reproducible peptide/protein ion yields in MALDI analysis, the sample preparation conditions, including identity of the matrix, matrix-to-analyte ratio, crystal size and homogeneity, presence of salts and buffers, peptide sequence, length, hydrophobicity, and complexity of the mixture have all been found to be significant to some degree [1-9].

Scanning electron microscopy, confocal laser scanning microscopy, X-ray crystallography, and MALDI imaging have been used to understand the relationship between analyte incorporation in the matrix, sample

Published online July 11, 2005

Address reprint requests to Dr. P. A. Limbach, 429K Rieveschl Laboratories for Mass Spectrometry, Department of Chemistry, University of Cincinnati, P.O. Box 210172, Cincinnati, OH 45221-0172, USA. E-mail: pat.limbach@uc.edu

* Present address: Mass Spectrometry and Proteomics Facility, 243 Fontana Laboratory, Ohio State University, Columbus, OH 43210. preparation, crystal morphology, and efficiency of MALDI ionization/desorption [10-14]. Dai et al. used confocal fluorescence microscopy to study variations in analyte distribution within the matrix crystals during various sample preparation methods [11]. They found that in a multi-component analysis, analytes were not evenly distributed in the matrix crystals even when homogeneous sample preparation methods like fast evaporation were used. Horneffer et al. used confocal laser scanning microscopy to study the analyte incorporation in the matrices 2,5-dihydroxy benzoic acid (2,5-DHB) and 2,6-dihydroxy benzoic acid (2,6-DHB) [10]. They reported that while analytes were incorporated in the crystals of 2,5-DHB matrix, the analytes were just attached to the surface of the crystals for the 2,6-DHB matrix. They correlated these results to a study by Gluckmann and coworkers which showed that the analyte desorbed by 2,5-DHB showed higher ion velocity and less fragmentation than the analyte desorbed from 2,6-DHB matrix [15].

Though surfactants have been used to improve the solubility of peptides and proteins in aqueous media [16], a number of reports demonstrate that the presence 
of surfactants, especially anionic surfactants like SDS, compromises both the signal-to-noise ratio and the mass accuracy of MALDI-MS analysis [17-20]. These limitations are presumed to arise due to poor incorporation of the protein in the crystalline matrix because the protein interacts with the surfactant more than with the matrix molecules [21]. Acid-labile surfactant (ALS) and ammonium dodecyl sulfate have been used for preparation of proteins and peptides analyzed by mass spectrometry [22-24]. Non-ionic and zwitterionic surfactants have been successfully used to analyze transmembrane proteins and protein digests for improved mass spectral analysis [25, 26].

Breaux et al. examined the effect of various cationic, anionic, zwitterionic and nonionic surfactants on mixtures of hydrophobic and hydrophilic peptides and found that SDS was successful in allowing detection of all components of the peptide mixture [27]. In a detailed examination of the effects of SDS on protein analysis by MALDI-MS, Amado et al. found that while the analyte signal deteriorates when SDS is present in small amounts ( 0 to $0.1 \% \mathrm{wt} / \mathrm{vol}$ ), the analyte signal was recovered at higher concentrations $(>0.3 \% \mathrm{wt} / \mathrm{vol})$ of SDS [21]. They also observed that this signal recovery was independent of the concentration or type of analyte. $\mathrm{Li}$ and coworkers have shown that various aspects like on-probe washing, choice of matrix, solvent, protein concentration, and sample preparation method affect the abundance of MALDI signals for SDS containing samples [28]. Using the two-layer sample preparation method, they established that 1\% SDS can be tolerated in peptide mapping experiments and 2\% SDS can be tolerated in the analysis of proteins [29].

As a follow-up to the work of Breaux et al., we previously reported preliminary findings showing that the addition of SDS led to an increase in sequence coverage during peptide mass fingerprinting of a standard proteins $[30,31]$. Our initial conclusion from that work was that SDS improved the homogeneity of the analyte:matrix crystals leading to improved sequence coverage. Here we report a more detailed investigation into the interactions between SDS micelles and peptide mixtures. We find that at surfactant concentrations near to the critical micelle concentration (CMC) of SDS $(0.23 \% \mathrm{wt} / \mathrm{vol})$, the number of peptides detected during MALDI-MS increases significantly. We attribute these findings to the differential solubility of peptides during cocrystallization with the matrix.

\section{Experimental}

\section{Materials}

Urea and ammonium bicarbonate, bovine cytochrome $c$, horse myoglobin, ovalbumin, bovine serum albumin (BSA), dithiothreotol (DTT), iodoacetamide (IAA), and ACTH and Angiotensin II from the ProteoMass peptide MALDI-MS calibration kit were obtained from Sigma (St. Louis, MO). Fluorescently labeled hydrophobic
(5-carboxyfluorescien)-VLAVAVLAVA and hydrophilic (5-carboxyfluorescien)-DEKRDREKRD peptides were obtained from SynPep Corporation (Dublin, CA). $\alpha$-Cyano-4-cinnamic acid (CHCA) was obtained from Fluka (Milwaukee, WI). Modified trypsin was obtained from Promega (Madison, MI). Sodium dodecyl sulfate (SDS) was obtained from Fischer Scientific (Fairlawn, NJ). $C_{18}$ Ziptip pipette tips were obtained from Millipore (Bedford, MA). HPLC grade solvents and acids were used for all experiments.

\section{Methods}

Tryptic digestion in urea buffer. Tryptic digestions were done using standard procedures [32]. ${ }^{\circ}$ Briefly, ${ }^{\circ}{ }^{\circ}$ solution of $8 \mathrm{M}$ urea and $0.4 \mathrm{M}$ ammonium bicarbonate $(\mathrm{pH}$ 7.5-8.5) was added to a $1 \mathrm{mg} / \mathrm{mL}$ aqueous protein solution. The solution was further diluted with water to yield a final concentration of $2 \mathrm{M}$ in urea. For BSA and ovalbumin, the proteins were reduced with $45 \mathrm{mM}$ DTT at $50{ }^{\circ} \mathrm{C}$ and alkylated with $100 \mathrm{mM}$ IAA at room temperature before dilution. The $0.1 \mathrm{mg} / \mathrm{mL}$ trypsin solution was prepared in $50 \mathrm{mM}$ acetic acid and added to the protein in a $1: 25$ (wt:wt) trypsin:protein ratio. Digestion was done at $37^{\circ} \mathrm{C}$ for $18 \mathrm{~h}$. Digested peptides were lyophilized to dryness and reconstituted in water to a final concentration of $0.5 \mathrm{mg} / \mathrm{mL}$.

Tryptic digestion in ammonium bicarbonate buffer. Tryptic digestions in ammonium bicarbonate were done using the same procedure as above with minor changes. Protein solutions were prepared in a $50 \mathrm{mM}$ ammonium bicarbonate ( $\mathrm{pH} 7.5-8.5)$ solution. BSA and ovalbumin were reduced and alkylated as before. The $0.1 \mathrm{mg} / \mathrm{mL}$ trypsin solution was prepared in $50 \mathrm{mM}$ ammonium bicarbonate and added to the protein in a 1:25 (wt:wt) trypsin:protein ratio. Digestion was done at $37^{\circ} \mathrm{C}$ for $18 \mathrm{~h}$. Digested peptides were lyophilized to dryness and reconstituted in water to a final concentration of 0.5 $\mathrm{mg} / \mathrm{mL}$.

Sample preparation. Aqueous solutions of SDS were prepared in concentrations of $0.01,0.02,0.05,0.1,0.2$, $0.3,0.5,1,5$ and $10 \%$ (wt/vol). A mixture of $5 \mathrm{pmol} / \mu \mathrm{L}$ of angiotensin II was mixed with $5 \mathrm{pmol} / \mu \mathrm{L}$ ACTH and $2 \mu \mathrm{L}$ of this mixture was added as internal calibrants to each $10 \mu \mathrm{L}$ aliquot of digested protein. Tryptic digests were combined with various concentrations of SDS in a 1:1 (vol:vol) ratio, vortexed for $15 \mathrm{~min}$, centrifuged and then vortexed again for $15 \mathrm{~min}$. The vortexing steps were found to be important for maximizing micellepeptide interactions.

Peptide fractionation. $\quad 20 \mu \mathrm{L}$ of BSA digest was reconstituted in $0.1 \%$ trifluoroacetic acid (TFA) at a concentration of $0.1 \mathrm{mg} / \mathrm{mL}$. The $10 \mu \mathrm{L} \mathrm{C18} \mathrm{Ziptip} \mathrm{pipette} \mathrm{tip} \mathrm{was}$ made wet with $50 \%$ acetonitrile in water and then equilibrated with $0.1 \%$ TFA. Once the peptides were loaded onto the Ziptip by 30 aspirations, the salt was 
Table 1. Percent amino acid sequence coverage from the MALDI analysis of tryptic peptides of four proteins $(0.1 \mu \mathrm{g}$ of digest per sample) after addition of SDS at different concentrations

\begin{tabular}{llccc}
\hline \multicolumn{1}{c}{ Proteins (buffer) } & $0 \% \mathrm{w} / \mathrm{v}$ SDS & $0.01-0.02 \% \mathrm{w} / \mathrm{v}$ SDS & $0.1-0.3 \% \mathrm{w} / \mathrm{v}$ SDS & $1 \% \mathrm{w} / \mathrm{v}$ SDS \\
\hline \hline Cytochrome $c$ (Bicarbonate) & $51 \%(53 / 104)^{\mathrm{a}}$ & $51 \%(53 / 104)$ & $76 \%(79 / 104)$ & $76 \%(79 / 104)$ \\
Cytochrome $c$ (Urea) & $42 \%(44 / 104)$ & $42 \%(44 / 104)$ & $63 \%(66 / 104)$ & $63 \%(66 / 104)$ \\
Myoglobin (Bicarbonate) & $67 \%(102 / 153)$ & $71 \%(108 / 153)$ & $94 \%(144 / 153)$ & $85 \%(131 / 153)$ \\
Myoglobin (Urea) & $84 \%(129 / 153)$ & $84 \%(129 / 153)$ & $96 \%(147 / 153)$ & $59 \%(91 / 153)$ \\
Ovalbumin (Bicarbonate) & $36 \%(140 / 385)$ & $36 \%(140 / 385)$ & $46 \%(179 / 385)$ & $35 \%(133 / 385)$ \\
Ovalbumin (Urea) & $12 \%(46 / 385)$ & $21 \%(82 / 385)$ & $31 \%(119 / 385)$ & N.D. \\
BSA (Bicarbonate) & $28 \%(167 / 607)$ & $35 \%(211 / 607)$ & $45 \%(276 / 607)$ & $23 \%(141 / 607)$ \\
BSA (Urea) & $14 \%(84 / 607)$ & $14 \%(84 / 607)$ & $18 \%(109 / 607)$ & N.D. \\
\hline
\end{tabular}

aThe numbers in parenthesis are the number of amino acids observed/total amino acids in that protein.

${ }^{b}$ N.D. - no mass spectral data of tryptic peptides could be detected.

washed off using $0.1 \%$ TFA. Five $\mu \mathrm{L}$ of elution buffer was taken in another Eppendorf tube and peptides were eluted off. The same Ziptip was washed with 5\% acetonitrile before the next elution was done. The different elution buffers were: $5 \%$ acetonitrile/ $0.1 \%$ TFA, $25 \%$ acetonitrile $/ 0.1 \%$ TFA, and $50 \%$ acetonitrile/ $0.1 \%$ TFA. Two $\mu \mathrm{L}$ of each eluted solution was mixed with the matrix or $0.3 \%(\mathrm{wt} / \mathrm{vol}) \mathrm{SDS}$ and matrix for MALDI analysis. To examine possible discrimination effects, $2 \mu \mathrm{L}$ of each eluted peptide solution was mixed together, then mixed with matrix or with $0.3 \%$ SDS and matrix, for further MALDI analysis.

MALDI-TOF analysis. All mass spectrometry experiments were done using a Bruker Reflex IV reflectron MALDI-TOF mass spectrometer (Bruker Daltonics, Billerica, MA) equipped with a nitrogen laser. Mass spectra were obtained in the positive-ion mode at an acceleration voltage of $20 \mathrm{kV}$. Each mass spectrum was the sum of 100 laser shots. The matrix solution consisting of $10 \mathrm{mg} / \mathrm{mL}$ CHCA in 50\% acetonitrile/0.1\% TFA was prepared fresh daily. Sample and matrix were combined in a 1:1 (vol:vol) ratio and immediately spotted on the target plate.

Fluorescence experiments. A $1.3 \mathrm{mg} / \mathrm{ml}$ stock solution of the labeled hydrophilic peptide was prepared in water and further diluted 1:18 (vol/vol) in water before analysis. $250 \mu \mathrm{L}$ of this solution was mixed with $250 \mu \mathrm{L}$ of water or $0.01,0.05,0.2,0.3,1$, and $5 \%$ SDS (wt/vol) solutions. For the hydrophobic peptide, the stock solution was prepared by dissolving $1 \mathrm{mg}$ of the labeled peptide in $20 \mu \mathrm{L}$ of TFA. The final concentration of the stock solution was brought to $1 \mathrm{mg} / \mathrm{mL}$ by adding 980 $\mu \mathrm{L}$ of $1 \% \mathrm{SDS}$ (wt/vol). One hundred twenty $\mu \mathrm{L}$ of the stock was diluted with water or appropriate concentration of SDS solution to obtain $400 \mu \mathrm{l}$ of final solutions containing $0.1,0.3,1$, and 3\% SDS (wt/vol). All solutions were sonicated for $10 \mathrm{~min}$. Fluorescence intensities of these solutions were measured using a Perkin Elmer (Boston, MA) LS 50B luminescence spectrometer at an excitation wavelength of $461 \mathrm{~nm}$ and an emission wavelength of $517 \mathrm{~nm}$ for the hydrophilic peptide and an excitation wavelength of $447 \mathrm{~nm}$ and an emission wavelength of $520 \mathrm{~nm}$ for the hydrophobic peptide.
Confocal microscopy. The solutions of labeled hydrophobic and hydrophilic peptide in water and $0.2 \%$ SDS were prepared exactly as discussed above. The purity of hydrophilic peptide was confirmed by HPLC and MALDI analysis. The purity of hydrophobic peptide was confirmed by surfactant-aided MALDI analysis. Various peptides solutions were mixed with matrix solution in a 1:1 (vol:vol) ratio and spotted on a glass slide. Fluorescence was monitored using Zeiss LSM 510 laser scanning confocal microscope (Zeiss Instrument. Thornwood, NY). A Plan-Neofluar 20X/0.75 UV objective was used for all experiments. The wavelengths for Channel 1 and Channel 2 were 543 and $488 \mathrm{~nm}$, respectively. Single scans with $1 \mu \mathrm{m}$ optical slices were used to obtain all images.

Surface tension studies. SDS solutions were prepared either in water or aqueous 5\% acetonitrile/0.1\% TFA solutions at concentrations ranging from 0 to $10 \%$ (wt/vol). The surface tension of these SDS solutions was determined by Du Nuoy Ring method using a Central Scientific Co. tensiometer (Chicago, IL). The instrument is calibrated with water (surface tension $=$ 72 dynes/cm).

\section{Results and Discussion}

\section{Effect of SDS on Peptide Mixtures}

Previously, we have found that the use of SDS at near-critical micelle concentrations tends to result in an increase in the number of peptides detected during MALDI-TOFMS $^{\circ}$ analysis ${ }^{\circ}$ of $^{\circ}$ tryptic $^{\circ}$ digests $^{\circ}\left[30,{ }^{\circ} 31\right]$. Here, a more comprehensive evaluation of the effects of SDS on MALDI-TOFMS analysis of peptide mixtures was undertaken. The purpose of these studies is to determine the rationale for such increases. Typical MALDI-TOFMS results from the analysis of tryptic digests of proteins in two different buffer systems, 50 $\mathrm{mM}$ ammonium bicarbonate and urea/ammonium bicarbonate, ${ }^{\circ}$ are $^{\circ}$ summarized $^{\circ}$ in $^{\circ}$ Table $^{\circ} 1 .^{\circ}$ The $^{\circ}$ tryptic peptides detected in these analyses could be grouped according to the concentration of SDS used in the sample $^{\circ}$ preparation $^{\circ}$ step. ${ }^{\circ}$ As $^{\circ}$ shown $^{\circ}$ in $^{\circ}$ Table $^{\circ} 1$, $^{\circ}$ the amino acid sequence coverage resulting from the de- 
Table 2. Tryptic peptides of cytochrome c digested in 50-mM ammonium bicarbonate buffer observed in MALDI analysis after addition of SDS at various concentrations; $0.1 \mu \mathrm{g}$ of digest per spot

\begin{tabular}{|c|c|c|c|c|c|c|}
\hline$m / z$ & Sequence & $0.00 \%$ & $0.02 \%$ & $0.10 \%$ & $0.20 \%$ & $1.00 \%$ \\
\hline 617.3 & KKGER & * & * & * & * & - \\
\hline 634.3 & IFVQK & - & - & * & * & * \\
\hline 678.3 & YIPGTK & - & - & * & * & - \\
\hline 964.5 & EDLIAYLK & * & * & * & * & - \\
\hline 1168.6 & TGPNLHGLFGR & * & * & * & * & * \\
\hline 1456.5 & TGQAPGFSYTDANK & * & * & * & * & * \\
\hline 1584.6 & KTGQAPGFSYTDANK & - & - & * & * & * \\
\hline 2009.9 & GITWGEETLMEYLENPK & * & * & * & * & * \\
\hline 2138.0 & GITWGEETLMEYLENPKK & * & * & * & * & * \\
\hline
\end{tabular}

*represents detected peptide.

tected tryptic peptides varied relative to the amount of SDS used in the sample preparation. In all cases, it was found that when SDS was added at a concentration near its $\mathrm{CMC}$, the number of peptides (i.e., sequence coverage) for all of the proteins investigated in this study increased rather significantly. As it has been shown that sample clean-up of tryptic peptides led to loss of some peptides, ${ }^{\circ}$ no $^{\circ}$ sample $^{\circ}$ desalting ${ }^{\circ}$ or $^{\circ}$ cleanup $^{\circ}$ steps ${ }^{\circ}$ were used to ensure that the effects of SDS could be monitored ${ }^{\circ}$ directly $[5,031,33]$.

The increase in sequence coverage was found to be independent of protein molecular weight. For example, myoglobin $(16 \mathrm{kDa})$ digested in the bicarbonate buffer yielded $67 \%$ sequence coverage in the absence of SDS. The same digest, when prepared with SDS near its CMC, yielded $94 \%$ sequence coverage. Similarly, BSA $(66 \mathrm{kDa})$ digested in bicarbonate buffer resulted in an increase in sequence coverage from 28 to $45 \%$ when prepared with SDS near its CMC. While Zhang and Li have found that the presence of lower concentrations of SDS $(<1 \%)$ does not affect tryptic digestion of proteins, they did not observe any trends relating to sequence coverage ${ }^{\circ}$ as $^{\circ} a^{\circ}$ function ${ }^{\circ}$ of $^{\circ} \operatorname{SDS}^{\circ}$ concentration ${ }^{\circ}[29] .{ }^{\circ} \mathrm{We}$ attribute the differences between these two findings to the sample preparation steps. SDS is added in the present study after tryptic digestion and the SDSpeptide mixture is vortexed thoroughly to ensure optimal peptide-micelle interactions. In our case, SDS is not merely present as a "contaminant" in the process, but as an integral component of the sample mixture.

Another general trend found during these analyses was related to the crystal morphology. At very low concentrations of SDS, the sequence coverage remained the same as if no SDS were added, although finding a sweet spot during analysis was more difficult. However, at very high concentrations ( $>1 \% \mathrm{wt} / \mathrm{vol}$ ) of SDS the formation of crystals was difficult, it took longer for the samples to dry and excess sodium in the sample led to a deterioration of spectral quality. These trends are similar ${ }^{\circ}$ to $^{\circ}$ those ${ }^{\circ}$ reported ${ }^{\circ}$ by $^{\circ} \mathrm{Zhang}^{\circ}$ and ${ }^{\circ} \mathrm{Li}^{\circ}[29]$.

We found that use of the bicarbonate buffer alone resulted in greater sequence coverage regardless of the amount of SDS added compared with digestions done in a urea/bicarbonate buffer, similar to that reported previously $^{\circ}\left[31,{ }^{\circ} 34\right] .{ }^{\circ}$ Table $^{\circ} 2^{\circ}$ shows $^{\circ}$ the ${ }^{\circ}$ results $^{\circ}$ from analysis of cytochrome $c$ digested with trypsin in ammonium bicarbonate buffer. After addition of $0.02 \%$ wt/vol SDS to the tryptic digest there was no difference in the number of detected peptides compared with the sample prepared without SDS, although the ease of finding a MALDI sweet spot was reduced. Addition of SDS up to $0.20 \% \mathrm{wt} / \mathrm{vol}$ increased the number of detected peptides, and it was noticed that the sample spot was more homogeneous. Further increases in the concentration of SDS added, yielded a reduction in detected peptides with no signal being observed at concentrations of $5 \% \mathrm{wt} / \mathrm{vol}$ or higher. These trends in numbers of peptides detected as a function of added SDS were reproducible, although some variation in peptide abundance $( \pm 25 \%)$ was noted during replicate analyses. ${ }^{\circ}$ Representative ${ }^{\circ}$ spectra ${ }^{\circ}$ are $^{\circ}$ shown ${ }^{\circ}{ }^{\circ}{ }^{\circ}$ Figure 1. Similar results were found for ovalbumin (data not shown), and these trends are comparable to those previously ${ }^{\circ}$ observed ${ }^{\circ}$ for $^{\circ}$ myoglobin $^{\circ}$ and $^{\circ} \mathrm{BSA}^{\circ}[31]$.

An examination of the increases in protein sequence coverage, when the amount of SDS is at or near the CMC, finds that the additional peptides detected for any of the proteins investigated cannot be classified by peptide hydrophobicity. For example, in the case of cytochrome $c$, the lower molecular weight, hydrophobic peptides YIPGTK (Bull and Breese Index [B and B\%]-46.5) and IFVQK (B and $\mathrm{B} \%-54.5)$ as well as the larger, hydrophilic peptide KTGQAPGFSYTDANK (B and $\mathrm{B} \%-26.6$ ) were observed exclusively after addition of 0.1 to $0.3 \%$ SDS. In the case of ovalbumin, the additional peptide ions observed were less than $1100 \mathrm{Da}$ and 5 of the 6 peaks observed had hydrophobicity Bull and Breese indices of over $35 \%$. At first glance, these results seem to suggest that micelles minimize differences in peptide hydrophobicity during the cocrystallization step.

\section{Interaction of SDS with Fluorescently Labeled Peptides}

To more fully appreciate the interactions between SDS micelles and hydrophobic and hydrophilic peptides, the behavior of fluorescently labeled peptides in solutions 
a)

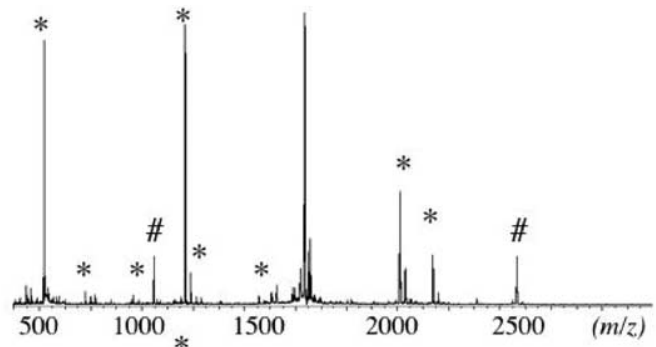

b)

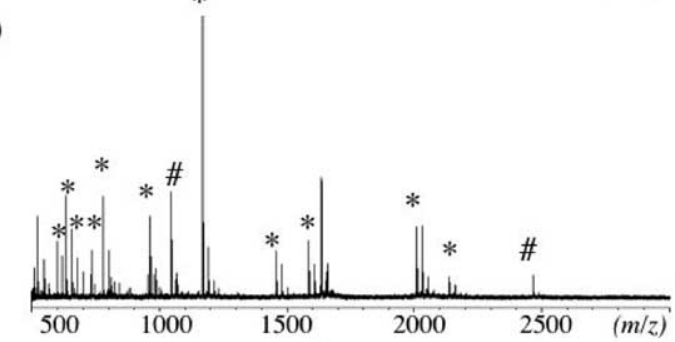

c)

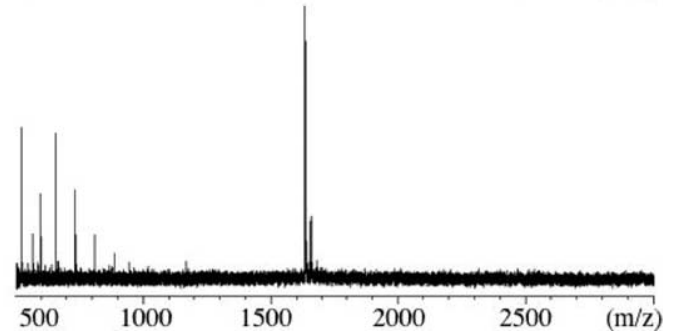

Figure 1. MALDI analysis of cytochrome $c$ digested with trypsin in 50-mM ammonium bicarbonate buffer with (a) no SDS, (b) $0.2 \%$ SDS, and (c) $5 \%$ SDS added before analysis. $0.1 \mu \mathrm{g}$ of digest per spot. Asterisks represent the peaks that match the theoretical tryptic digest of cytochrome $c$ and the number signs represent the internal calibrants.

of SDS was investigated. If SDS micelles improve the solubility of hydrophobic peptides as assumed previously $^{\circ}\left[27,{ }^{\circ} 30,{ }^{\circ} 35\right]^{\circ}$, then $^{\circ}$ one $^{\circ}$ would $^{\circ}$ predict $^{\circ}$ to ${ }^{\circ}$ see $^{\circ}$ an increase in fluorescence once the CMC of SDS is reached. ${ }^{\circ} \mathrm{As}^{\circ}$ seen $^{\circ}$ in $^{\circ}$ Figure $^{\circ} 2 \mathrm{a}^{\circ},{ }^{\circ}$ the ${ }^{\circ}$ fluorescence ${ }^{\circ}$ of ${ }^{\circ}$ the hydrophobic peptide, (5-carboxyfluorescein)-VLAVAVLAVA, increases significantly at SDS concentrations above the CMC and then plateaus at even higher SDS concentrations. Therefore, apparently SDS serves to increase the solubility of hydrophobic peptides in aqueous solutions.

Similar experiments were done using a fluorescently labeled hydrophilic peptide, (5-carboxyfluorescein)DEKRDREKRD $^{\circ}$ (Figure ${ }^{\circ} 2 \mathrm{~b}$ )..$^{\circ}$ In $^{\circ}$ contrast $^{\circ}$ to $^{\circ}$ the ${ }^{\circ}$ results found for the hydrophobic peptide, initially the fluorescence decreased upon addition of SDS. This decrease in fluorescence is attributed to interactions between the anionic SDS and the basic peptide. At concentrations above the $\mathrm{CMC}$, fluorescence intensity is recovered, suggesting aggregation among the SDS molecules instead of between SDS and the hydrophilic peptide. At the highest concentrations of SDS investigated, the fluorescence is appreciably higher for the labeled peptide than when it is in aqueous solution. This increase is likely attributable to aggregation between the micelles and ${ }^{\circ}$ the $^{\circ}$ labeled ${ }^{\circ}$ peptide ${ }^{\circ}[35]$.

\section{Solvent Effects on Micelle Formation}

To ensure that micelles of SDS formed in the solutions commonly used to prepare peptides for MALDI-MS, surface tension measurements for SDS were obtained. The solvent conditions used to prepare samples for MALDI-MS do not significantly affect the CMC of SDS (data not shown) as expected. Thus, when SDS is present at concentrations above $0.2 \% \mathrm{wt} / \mathrm{vol}$, micelles are present after vortexing and are assumed to be affecting the MALDI-MS results for peptide mixtures.

\section{Effect of SDS on Fluorescently Labeled Peptides in Matrix Crystals}

To understand the effect of SDS on the hydrophobic and hydrophilic peptides in matrix crystals, further experiments were performed. The samples were prepared in exactly the same way as for MALDI analysis using the dried droplet method and spotted on a glass slide. Confocal microscopy images of the matrix, matrix with SDS, hydrophobic peptide with and without SDS in matrix, and hydrophilic peptide with and without

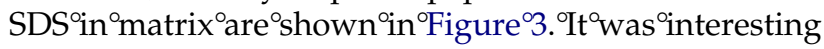
to note that the morphology of the crystals of CHCA in the presence and absence of SDS remain the same although there is considerable aggregation among the matrix ${ }^{\circ}$ crystals ${ }^{\circ}$ in $^{\circ}$ the ${ }^{\circ}$ presence ${ }^{\circ}{ }^{\circ}{ }^{\circ} S^{\circ} S^{\circ}{ }^{\circ}{ }^{\circ}$ seen $^{\circ}{ }^{\circ}{ }^{\circ}$ Figure $3 \mathrm{a}^{\circ}$ and $^{\circ} 3 \mathrm{~b} .^{\circ}{ }^{\circ}$ The $^{\circ}$ matrix $^{\circ}$ does $^{\circ}$ not $^{\circ}$ fluoresce $^{\circ}$ in $^{\circ}$ the ${ }^{\circ}$ green
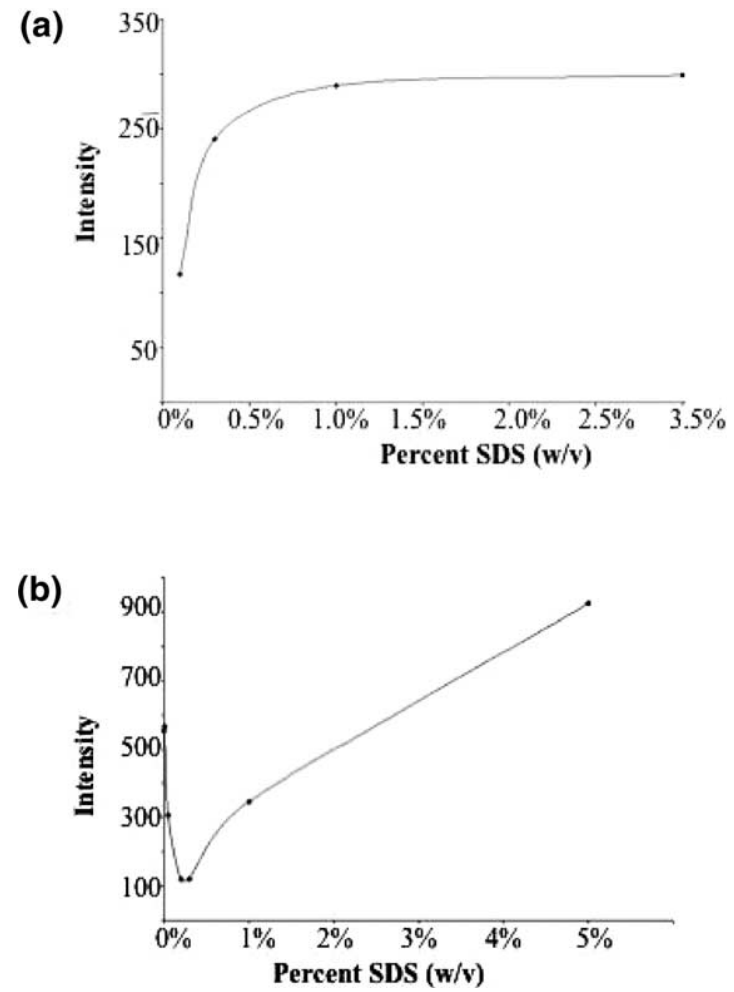

Figure 2. Fluorescence intensities of two labeled peptides (a) (5-carboxyfluorescien)-VLAVAVLAVA and (b) (5-carboxyfluorescien)-DEKRDREKRD as a function of added SDS. 

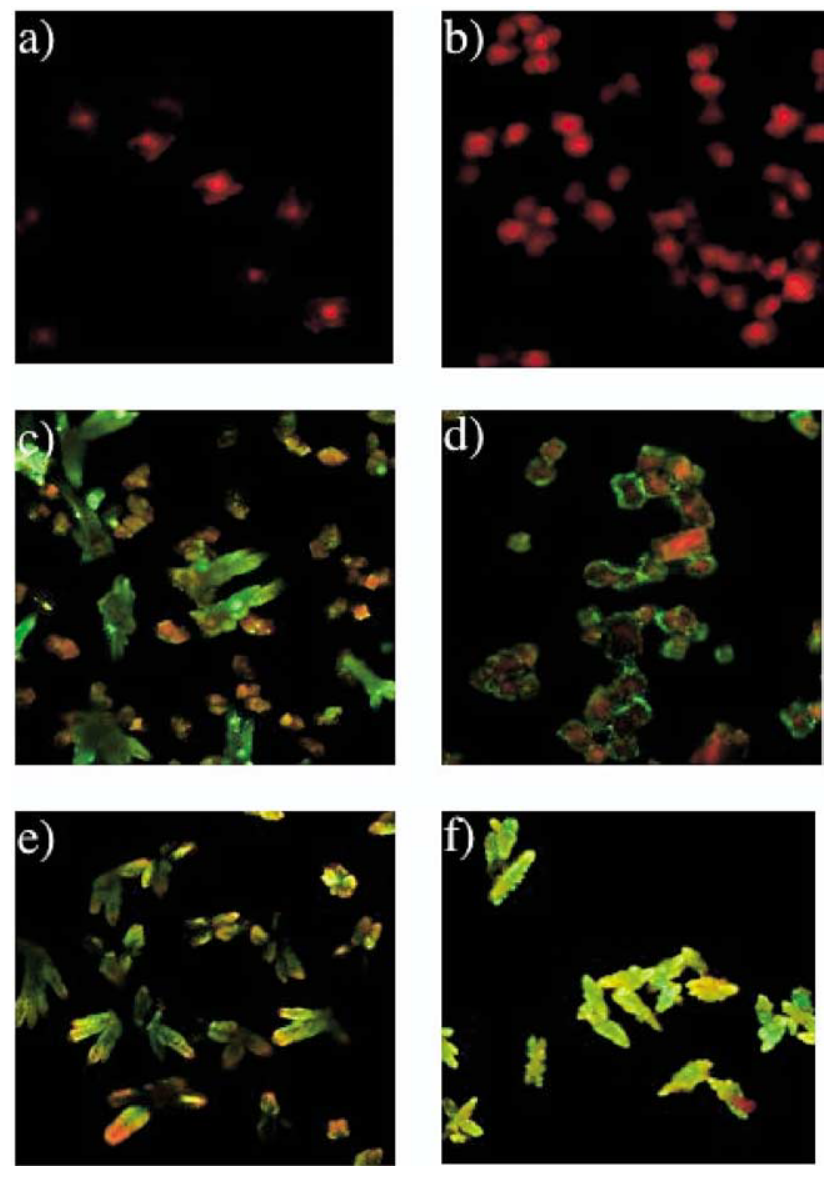

Figure 3. Confocal microscopy of CHCA matrix crystals obtained by superimposing the transmission phase-contrast images of matrix crystals (in red) and analyte (in green). (a) matrix with no additives, (b) matrix with $0.2 \%$ SDS, (c) matrix with hydrophobic peptide, (5-carboxyfluorescien)-VLAVAVLAVA, (d) matrix with $0.2 \%$ SDS and hydrophobic peptide, (e) matrix with hydrophilic peptide, (5-carboxyfluorescien)-DEKRDREKRD, and (f) matrix with $0.2 \%$ SDS and hydrophilic peptide.

channel and will not interfere in the study of the fluorescence ${ }^{\circ}$ properties ${ }^{\circ}$ of ${ }^{\circ}$ the ${ }^{\circ}$ tagged ${ }^{\circ}$ peptides. ${ }^{\circ}$ Figure $3 \mathrm{c}^{\circ}$ is ${ }^{\circ}$ the ${ }^{\circ}$ confocal $^{\circ}$ image $^{\circ}$ of $^{\circ}$ the ${ }^{\circ}$ hydrophobic ${ }^{\circ}$ peptide mixed with the matrix. There is a clear heterogeneity both in crystal morphology as well as fluorescence in this sample. The (5-carboxyfluorescein)-VLAVAVLAVA hydrophobic peptide forms bright green clusters attributable to the 5-carboxy-fluorescien tag, while the small cubic crystals attributable to matrix are observed in red. This image reinforces the hypothesis that hydrophobic peptides do not cocrystallize with the matrix. On the other hand, the presence of SDS in the hydrophobic peptide solution seems to improve the cocrystallization of $^{\circ}$ the ${ }^{\circ}$ matrix ${ }^{\circ}$ and $^{\circ}$ the $^{\circ}$ peptide. $^{\circ}$ As $^{\circ}$ seen $^{\circ}$ in ${ }^{\circ}$ Figure $^{\circ} 3 \mathrm{~d}$, crystal shapes are more uniform with brighter green, attributable to the labeled peptide, on the edges and completely red, attributable to the matrix, in the inner side of the crystals.

The (5-carboxyfluorescein)-DEKRDREKRD hydrophilic peptide, on the other hand, cocrystallized with the ${ }^{\circ}$ matrix $^{\circ}$ as $^{\circ}$ observed ${ }^{\circ}$ in $^{\circ}$ Figure $^{\circ} 3 e^{\circ}{ }^{\circ}$ When $^{\circ}$ SDS $^{\circ}$ was added to this peptide and then crystallized with the matrix, not only was there a reduction in fluorescence intensity but also the crystal edges were not as sharp as

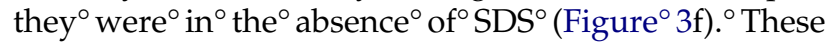
results reaffirm that on addition of SDS at its CMC to the fluorescently labeled hydrophilic peptide, there is a decrease in fluorescence due to micelle-peptide interactions.

\section{Analyte Discrimination in MALDI-MS Analysis}

Although the previous experiments demonstrate that micelle formation occurs at SDS concentrations above the CMC, that these micelles minimize differences in peptide solubility, and that the peptides detected from tryptic digests of model proteins differ in the presence and absence of SDS, another factor that can play a role in influencing the detected peptides is the inherent discrimination effects seen during MALDI-MS of complex mixtures. Burkitt and coworkers previously reported that MALDI results obtained from fractionated peptide digests are not equivalent to MALDI results obtained ${ }^{\circ}$ from $^{\circ}$ unfractionated ${ }^{\circ}$ peptide ${ }^{\circ}$ digests $^{\circ}[5]$. Thus, additional studies were conducted here to determine to what extent differences in MALDI data obtained in the absence and presence of SDS micelles are due exclusively ${ }^{\circ}$ to $^{\circ}$ micelle $^{\circ}$ formation ${ }^{\circ}$ versus ${ }^{\circ}$ those ${ }^{\circ}$ due ${ }^{\circ}$ simply ${ }^{\circ}$ to sample fractionation.

Table $^{\circ} 3^{\circ}$ contains $^{\circ}$ the ${ }^{\circ}$ results ${ }^{\circ}$ of ${ }^{\circ}$ MALDI-MS ${ }^{\circ}$ analyses of tryptic digests of BSA that were fractionated by $\mathrm{C} 18$ ZipTips and various concentrations of organic solvent. For each elution condition, half of the eluent was analyzed directly and the other half was combined with $0.3 \% \mathrm{wt} / \mathrm{vol}$ SDS before analysis. For comparison, eluents from each solvent were also combined before MALDI-MS. ${ }^{\circ}$ Further, ${ }^{\circ}$ Table $^{\circ} 3^{\circ}$ also $^{\circ}$ contains $^{\circ}$ peptides detected by MALDI-MS for unfractionated BSA tryptic digests.

Examination ${ }^{\circ}$ of ${ }^{\circ}$ the ${ }^{\circ}$ data $^{\circ}{ }^{\circ}{ }^{\circ}$ Table $^{\circ} 3^{\circ}$ reveals ${ }^{\circ}$ distinct differences between eluted fractions analyzed in the absence and presence of SDS micelles. The peptides detected from $5 \%$ acetonitrile elution (presumably the most hydrophilic peptides in the digestion mixture) and no SDS micelles all had Bull and Breese indices below $35 \%$. The 25 and $50 \%$ acetonitrile elutions with no SDS micelles all had B and B indices above $35 \%$ with one exception. As previously observed by Derrick and coworkers, peptides detected after fractionation correlate well with the elution conditions when no SDS is present [5]. ${ }^{\circ}$ However, ${ }^{\circ}$ after $^{\circ}$ combining $^{\circ}$ each $^{\circ}$ acetonitrile ${ }^{\circ}$ eluent, more hydrophobic components were detected suggesting matrix:analyte incorporation effects led to the MALDI responses observed here.

Distinctly different results were obtained when the acetonitrile eluents were prepared with SDS micelles before MALDI analysis. Although the 5\% acetonitrile fraction with SDS yielded peptides having B and B indices less than $40 \%$, the 25 and $50 \%$ acetonitrile 
Table 3. Tryptic peptide fragments of bovine serum albumin digested in 50-mM ammonium bicarbonate buffer observed in MALDI analysis after fractionation using C18 ZipTips and the described solvent

\begin{tabular}{|c|c|c|c|}
\hline Fragments & Hydrophobicity BB\% & Fragments & Hydrophobicity BB\% \\
\hline \multicolumn{2}{|c|}{$5 \%$ ACN $/ 0.1 \%$ TFA } & \multicolumn{2}{|c|}{$5 \%$ ACN $/ 0.1 \%$ TFA with SDS } \\
\hline $76-88$ & 20.9 & $76-88$ & 20.9 \\
\hline $267-280$ & 27.7 & $413-420$ & 21.5 \\
\hline 184-197 & 27.9 & $460-468$ & 22.5 \\
\hline $375-386$ & 31.8 & $499-507$ & 32.3 \\
\hline $387-399$ & 34.7 & $123-130$ & 36.3 \\
\hline $286-297$ & 34.8 & $139-151$ & 39.9 \\
\hline \multicolumn{2}{|c|}{$25 \%$ ACN $/ 0.1 \%$ TFA } & \multicolumn{2}{|c|}{$25 \%$ ACN/0.1\% TFA with SDS } \\
\hline $508-523$ & 40.9 & $139-151$ & 20.9 \\
\hline $421-433$ & 46.3 & $106-117$ & 27.4 \\
\hline \multirow[t]{7}{*}{$347-359$} & 52.1 & $267-280$ & 27.7 \\
\hline & & 387-399 & 34.7 \\
\hline & & $508-523$ & 40.9 \\
\hline & & $401-412$ & 42.5 \\
\hline & & $89-100$ & 44.4 \\
\hline & & $66-75$ & 45.8 \\
\hline & & $421-433$ & 46.3 \\
\hline \multicolumn{2}{|c|}{$50 \%$ ACN $/ 0.1 \%$ TFA } & \multicolumn{2}{|c|}{$50 \%$ ACN $/ 0.1 \%$ TFA with SDS } \\
\hline $106-117$ & 27.4 & $106-117$ & 27.4 \\
\hline $508-523$ & 40.9 & $375-386$ & 31.8 \\
\hline $421-433$ & 46.3 & 387-399 & 34.7 \\
\hline $45-65$ & 46.4 & $508-523$ & 40.9 \\
\hline $469-482$ & 50.4 & $66-75$ & 45.8 \\
\hline \multirow[t]{2}{*}{$347-359$} & 52.1 & $421-433$ & 46.3 \\
\hline & & $347-359$ & 52.1 \\
\hline Combined & & \multicolumn{2}{|c|}{ Combined with SDS } \\
\hline $508-523$ & 40.9 & $387-399$ & 34.7 \\
\hline $402-412$ & 42.4 & $508-523$ & 40.9 \\
\hline $529-544$ & 44.8 & 89-100 & 44.4 \\
\hline $66-75$ & 45.8 & $421-4335$ & 46.3 \\
\hline $421-433$ & 46.3 & $347-359$ & 52.1 \\
\hline $469-482$ & 50.4 & & \\
\hline $347-359$ & 52.1 & & \\
\hline \multicolumn{2}{|c|}{ No fractionation } & \multicolumn{2}{|c|}{ No fractionation with SDS } \\
\hline $76-88$ & 20.9 & $76-88$ & 20.9 \\
\hline $267-280$ & 27.7 & $267-280$ & 27.7 \\
\hline $123-130$ & 36.3 & $310-318$ & 31.3 \\
\hline $66-75$ & 45.8 & $375-386$ & 31.8 \\
\hline $469-482$ & 50.4 & 387-399 & 34.7 \\
\hline \multirow[t]{7}{*}{$347-359$} & 52.1 & $123-130$ & 36.3 \\
\hline & & $402-412$ & 42.4 \\
\hline & & $529-544$ & 44.8 \\
\hline & & $66-75$ & 45.8 \\
\hline & & $421-433$ & 46.3 \\
\hline & & $469-482$ & 50.4 \\
\hline & & $347-359$ & 52.1 \\
\hline
\end{tabular}

fractions yielded peptides of widely varying B and B indices. Because SDS was added after elution from the ZipTip, these results suggest that incorporation into the MALDI crystalline matrix is affected by the presence of SDS micelles. For the higher organic elutions, more peptides were detected when SDS micelles were present than in their absence. Moreover, because these peptides have widely varying B and B indices, specific suppression effects due to peptide chemical composition do not appear to be evident. Rather, these data suggest that during the sample spotting/evaporation process the more hydrophilic peptides are excluded from the matrix crystals when SDS is not present. This interpretation is supported by results from Amado and coworkers who observed similar exclusion of hydrophilic amino acids and small peptides during MALDI analysis ${ }^{\circ}[1] .^{\circ}$ In $^{\circ}$ contrast $^{\circ}$ the ${ }^{\circ}$ presence $^{\circ}$ of $^{\circ}$ SDS $^{\circ}$ micelles minimizes exclusion effects leading to more representative incorporation of peptides within the matrix crystals.

Most surprising are the results obtained when the BSA digest is not fractionated before MALDI-MS analysis. As noted above, a significantly larger fraction of tryptic peptides are detected from unfractionated mixtures when SDS micelles are present. However, only $50 \%$ of the peptides detected in the unfractionated 
analysis were detected in any of the fractionated analyses, supporting prior evidence that signal suppression is present during MALDI analysis of complex mixtures $[1, \circ 5, \circ, \circ 36]$.

\section{Net Effect of SDS}

While the data from this study cannot point to a specific interpretation of the effect of SDS micelles on the resulting peptides detected by MALDI-MS, a number of interesting observations can be made. The surface tension studies demonstrate that micelles are formed under the experimental conditions employed here. The fluorescence and confocol microscopy studies find that hydrophobic peptides are solubilized in the presence of micelles and that these peptides cocrystallize more effectively with the matrix. The mass spectral data finds that a greater number of peptides, irrespective of peptide hydrophobicity, are detected in the presence of SDS micelles than in their absence when all other variables are held constant. Finally, the fractionation studies also confirm that a broader range of hydrophobicities are detected in the presence of SDS micelles.

As noted by a reviewer, these findings are in contrast to those of $\mathrm{Li}$ and coworkers, who optimized the two-layer method for analyzing proteins solubilized in the ${ }^{\circ}$ presence $^{\circ}$ of $^{\circ} \operatorname{SDS}^{\circ}[28] .{ }^{\circ} \mathrm{As}^{\circ}$ the $^{\circ}$ present $^{\circ}$ study $^{\circ}$ was conducted by spotting samples using the dried-droplet method, our results suggest that micelle-peptide interactions affect matrix-analyte cocrystallization in a manner somewhat dependent upon this spotting technique. Our hypothesis is that as the solvent starts to evaporate after spotting on the MALDI target plate, SDS micelles with the interacting peptides will precipitate and reach the surface of the crystal lattice. This precipitation step improves the homogeneity of the sample crystals, delivers more peptides to the surface of the droplet for incorporation within the matrix, and leads to an increase in the number of detected peptides. This effect would become especially significant for the dried-droplet method of sample preparation, where microscopy studies have shown peripheral deposition of hydrophilic analytes during sample drying process attributableqo Marangoni ${ }^{\circ}$ effects $[1]$. Because of the interactions of the peptides with SDS micelles, there seems to be a decrease in these Marangoni effects, thus reducing the formation of nonhomogeneous crystals within a narrow range $(0.1-0.3 \%)$ of SDS concentrations. As more peptides are delivered to the surface of the droplet, MALDI discrimination effects due to analyte interactions such as $^{\circ}$ those ${ }^{\circ}$ reported $^{\circ}$ by $^{\circ}$ Derrick $^{\circ}$ and $^{\circ}$ coworkers $^{\circ}[5]^{\circ}$ can again become significant. If correct, additional peptides being detected in the presence of micelles would not be correlated to hydrophobicity, and significant differences would be seen between fractionated and unfractionated samples. Such explanations are supported by the data obtained here, but further studies will be necessary to confirm this hypothesis.

\section{Conclusions}

The addition of SDS at critical micellar concentrations leads to an increase in the number of tryptic peptides detected during MALDI-MS analysis of proteolytic digests. It has been found that under the experimental conditions used in this work, SDS micelles form in the MALDI sample solution. Fluorescence studies demonstrate that these micelles interact with both hydrophilic and hydrophobic peptides, although the interaction is presumed to differ between hydrophilic and hydrophobic $^{\circ}$ analytes. ${ }^{\circ}$ Fractionation ${ }^{\circ}$ studies $^{\circ}$ were $^{\circ}$ conducted $^{\circ}$ to determine the extent sample suppression and analyte hydrophobicity affect MALDI-MS results. These fractionation studies clearly show that analyte hydrophobicity is not the determining factor in increased sequence coverage from complex peptide mixtures analyzed in the presence of SDS. Further, while sample suppression is obviously a factor to be accounted for during MALDI analysis of complex mixtures, analyte incorporation within the matrix appears to be as significant and is affected by the presence of SDS micelles. This is especially true for the dried-droplet method, which is known to result in inhomogeneous matrixanalyte crystals. These results further support prior conclusions that limitations to MALDI-MS analysis of complex mixtures arise from many experimental variables including analyte heterogeneity and improvements to the analyte:matrix cocrystallization process should yield higher quality MALDI mass spectral data.

\section{Acknowledgments}

The authors gratefully acknowledge financial support of this work provided by an American Society for Mass Spectrometry Research Award sponsored by Finnigan and the University of Cincinnati.

\section{References}

1. Amado, F. M. L.; Domingues, P.; Santana-Marques, M. G.; Ferrer-Correia, A. J.; Tomer, K. B. Discrimination effects and sensitivity variations in matrix-assisted laser desorption/ionization. Rapid Commun. Mass Spectrom. 1997, 11, 1347-1352.

2. Krause, E.; Wenschuh, H.; Jungblut, P. R. The dominance of arginine-containing peptides in MALDI-derived tryptic mass fingerprints of proteins. Anal. Chem. 1999, 71, 4160-4165.

3. Valero, M.-L.; Giraltz, E.; Andreu, D. An investigation of residue-specific contribution to peptide desorption in MALDITOF mass spectrometry. Lett. Pept. Sci. 1999, 6, 109-115.

4. Belghazi, M.; Bathany, K.; Hountondji, C.; Grandier-Vazeille, X.; Manon, S.; Schmitter, J.-M. Analysis of protein sequences and protein complexes by matrix-assisted laser desorption/ ionization mass spectrometry. Proteomics 2001, 1, 946-954.

5. Burkitt, W. I.; Giannakopulos, A. E.; Sideridou, F.; Bashir, S.; Derrick, P. J. Discrimination effects in MALDI-MS of mixtures of peptides- analysis of the proteome. Aust. J. Chem. 2003, 56, 369-377.

6. Vorm, O.; Mann, M. Improved mass accuracy in matrixassisted laser desorption/ionization time-of-flight mass spectrometry of peptides. J. Am. Soc. Mass Spectrom. 1994, 5, 955-958. 
7. Cohen, S. L.; Chait, B. T. Influence of matrix solution conditions on the MALDI-MS analysis of peptides and proteins. Anal. Chem. 1996, 68, 31-37.

8. Sze, E. T. P.; Chan, D. T.-W.; Wang, G. Formulation of matrix solutions for use in matrix-assisted laser desorption/ionization of biomolecules. J. Am. Soc. Mass Spectrom. 1998, 9, 166-177.

9. Yao, J.; Scott, J. R.; Young, M. K.; Wilkins, C. L. Importance of matrix analyte ratio for buffer tolerance using 2,5-dihydroxy benzoic acid as matrix in matrix-assisted laser desorption/ ionization Fourier transform mass spectrometry and matrixassisted laser desorption/ionization time-of-flight. J. Am. Soc. Mass Spectrom. 1998, 9, 805-813.

10. Horneffer, V.; Forsmann, A.; Strupat, K.; Hillenkamp, F.; Kubitscheck, U. Localization of analyte molecules in MALDI preparations by confocal laser scanning microscopy. Anal. Chem. 2001, 73, 1016-1022.

11. Dai, Y.; Whittal, R. M.; Li, L. Confocal fluorescence microscopic imaging for investigating the analyte distribution in MALDI matrices. Anal. Chem. 1996, 68, 2494-2500.

12. Allwood, D. A.; Perera, I. K.; Perkins, J.; Dyer, P. E.; Oldershaw, G. A. Preparation of near homogeneous samples for analysis of matrix-assisted laser desorption/ionization processes. Appl. Surf. Sci. 1996, 103, 231-244.

13. Garden, R. W.; Sweedler, J. V. Hetrogeneity within MALDI samples as revealed by mass spectrometric imaging. Anal. Chem. 2000, 72, 30-36.

14. Strupat, K.; Kampmeier, J.; Horneffer, V. Investigation of 2,5-DHB and succinic acid as matrices for UV and IR MALDI. Part II . Crystallographic and mass spectrometric analysis. Int. J. Mass Spectrom. Ion Processes 1997, 169/170, 43-70.

15. Gluckmann, M.; Karas, M. The initial velocity and its dependence on matrix, analyte, and preparation method in ultraviolet matrix assisted laser desorption/ionization. J. Mass Spectrom. 1999, 34, 467-477.

16. Jones, M. N. Surfactant interactions with biomembranes and proteins. Chem. Soc. Rev. 1992, 21, 127-136.

17. Lahm, H.-W.; Langen, H. Mass spectrometry: A tool for the identification of proteins separated by gels. Electrophoresis 2000, 21, 2105-2114.

18. Rosinke, B.; Strupat, K.; Hillenkamp, F.; Rosenbusch, J.; Dencher, N.; Kruger, U.; Galla, H. Matrix-assisted laser desorption/ionization mass spectrometry (MALDI-MS) of membrane proteins and noncovalent complexes. J. Mass Spectrom. 1995, 30, 1462-1468.

19. Schnaible, V.; Michels, J.; Zeth, K.; Freigang, J.; Welte, W.; Buhler, S.; Glocker, M. O.; Przybylski, M. Approaches to the characterization of membrane channel proteins (porins) by UV MALDI-MS. Int. J. Mass Spectrom. Ion Processes 1997, 169, 165-177.

20. Walker, A. K.; Wu, Y.; Timmons, R. B.; Kinsel, G. R.; Nelson, K. D. Effects of protein-surface interactions on protein ion signals in MALDI mass spectrometry. Anal. Chem. 1999, 71, $268-272$.

21. Amado, F. M. L.; SantanaMarques, M. G.; Ferrer-Correia, A. J.; Tomer, K. B. Analysis of Peptide and Protein Samples Containing Surfactants by MALDI-MS. Anal. Chem. 1997, 69, 1102-1106.
22. Konig, S.; Schmidt, O.; Rose, K.; Thanos, S.; Besselmann, M.; Zeller, M. Sodium dodecyl sulfate versus acid-labile surfactant gel electrophoresis: Comparative proteomic studies on rat retina and mouse brain. Electrophoresis 2003, 24, 751-756.

23. Meng, F.; Cargile, B. J.; Patrie, S. M.; McLoughlin, S. M.; Keller, N. L. Processing complex mixtures of intact proteins for direct analysis by mass spectrometry. Anal. Chem. 2002, 74, 2923 2929.

24. Zhang, N.; Li, L. Ammonium dodecyl sulfate as an alternative to sodium dodecyl sulfate for protein sample preparation with improved performance in MALDI mass spectrometry. Anal. Chem. 2002, 74, 1729-1736.

25. Cadene, M.; Chait, B. T. A robust, detergent-friendly method for mass spectrometric analysis of integral membrane proteins. Anal. Chem. 2000, 72, 5655-5658.

26. Barnidge, D. R.; Dratz, E. A.; Jesaitis, A. J.; Sunner, J. Extraction method for analysis of detergent-solubilized bacteriorhodopsin and hydrophobic peptides by electrospray ionization mass spectrometry. Anal. Biochem. 1999, 269, 1-9.

27. Breaux, G. A.; Green-Church, K. B.; France, A.; Limbach, P. A. Surfactant-aided, matrix-assisted laser desorption/ionization mass spectrometry of hydrophobic and hydrophilic peptides. Anal. Chem. 2000, 72, 1169-1174.

28. Zhang, N.; Doucette, A.; Li, L. Two-layer sample preparation method for MALDI mass spectrometric analysis for protein and peptide samples containing sodium dodecyl sulfate. Anal. Chem. 2001, 73, 2968-2975.

29. Zhang, N.; Li, L. Effects of common surfactants on protein digestion and matrix-assisted laser desorption/ionization mass spectrometric analysis of the digested peptides using two-layer sample preparation. Rapid Commun. Mass Spectrom. 2004, 18, 889-896.

30. Tummala, R.; Ballard, L. M.; Breaux, G. A.; Green-Church, K. B.; Limbach, P. A. Investigation into surfactant-aided matrix assisted laser desorption/ionization-mass spectrometry for peptide mapping. Proceedings of SPIE, January 2000, San Jose, California, pp 56-60.

31. Tummala, R.; Limbach, P. A. Effect of sodium dodecyl sulfate micelles on peptide mass fingerprinting by matrix-assisted laser desorption/ionization mass spectrometry (MALDI-MS). Rapid Commun. Mass Spectrom. 2004, 18, 2031-2035.

32. Stone, K. L.; Williams, K. R. Enzymatic digestion of proteins in solution and in SDS polyacrylamide gels. The Protein Protocol Handbook; Totowa, NJ: Humana Press, pp 415-425.

33. Fountoulakis, M.; Langen, H. Identification of proteins by matrix-assisted laser desorption ionization-mass spectrometry following in-gel digestion in low-salt, nonvolatile buffer and simplified peptide recovery. Anal. Biochem. 1997, 250, 153-156.

34. Doucette, A.; Craft, D.; Li, L. Protein concentration and enzyme digestion on microbeads for MALDI-TOF peptide mass mapping of proteins from dilute solutions. Anal. Chem. 2000, 72, 3355-3362.

35. Orfi, L.; Lin, M.; Larive, C. K. Measurement of SDS micellepeptide association using $1 \mathrm{H}$ NMR chemical shift analysis and pulsed-field gradient NMR spectroscopy. Anal. Chem. 1998, 39, 1339-1345.

36. Wang, M. Z.; Fitzgerald, M. C. A solid sample preparation method that reduces signal suppression effects in the MALDI analysis of peptides. Anal. Chem. 2001, 73, 625-631. 